\title{
SAGGIO SULLA FILOSOFIA ESTETICA: APPUNTAMENTI SEMIOTICI SULLA REALTÀ ESTETICA
}

\author{
Eduardo Carlos Bianca Bittar \\ Aluno do Curso de Graduação da Faculdade de Direito da USP
}

\begin{abstract}
Sumario:
La pluralità e la complexità del cappolavoro artistico chiedono un'analise che non sia ridotta ai punti di vista teorici più tradizionali. Tutta la struttura dell'opera estetica indica la necessità di vedere i fenomeni estetici come fenomeni semiotici, il cui si trova nell'interiore delle considerazione culturalistiche più ampie. Così, filosofia estetica e semiotica hanno diversi punti di collegamento a essere scoperti, lavoro che può essere cominciato con la ricerca sui segni estetici.
\end{abstract}

\begin{abstract}
:
The plurality and the complexity of artistic masterpieces demand an analysis that can not be reduced to the most traditional theoretic points of view. All the structure of aesthetic works creates the need for aesthetic phenomena to be seen as semiotic phenomena, within the borders of deeper cultural remarks. Therefore, aeshetic philosophy and semiotics have many linking points yet to be found. The study of aesthetic signs could be the starting point of this research.
\end{abstract}

Unitermos: Filosofia estética e semiótica.

Sumario

1. Artificio umano e natura

2. La formazione dell'opera estetica

3. La estetica ed i segni estitici

4. Aspetti interpretativi dell'opera estetica

Bibliografia 
1. Artificio umano e natura

La scoperta di segni estetici nel centro della vita sociale è propriamente l'azione di definire le ragione di esistenza della comunicazione in tutte le manifestazione culturale umane. Non si esclude mai l'interpretazione dei fenomeni culturali come produttivi di segni $^{1}$-- essendo che questi sono doppiamente convenzionali, tanto come significanti, quanto come significati, dal punto di vista della relazione intrinseca che questi due elementi hanno --, che dimostrano l'intervento della ragione sulla natura, dell'uomo sull'universo naturale. Dunque, c'è propriamente qui una relazione diretta tra le idee di produzione segnica e attività culturale umana. La cultura è così importante nel seno della vita sociale come fenomeno antropologico, e indica la capacità di intervenire nel piano della naturalità, e non soltanto questo, ma la capacità di perfezionare queste attività, di trasformarle $\mathrm{e}$ di trasferirle come legato alle altre generazione future. ${ }^{2}$ L'idea è proprio quella di un processo che non finisce mai, anzi, si produce e riproduce, generazione dopo generazione, di acquiste in acquiste ad infinitum; questo non può essere fermato, ed i suoi risultati non possono essere previsti, datta l'impossibilità di prevedersi i risultati delle azioni culturali. Insomma, si può dire non soltanto che la cultura partecipa della nozione di sociabilità e di manifestazione comunicativa, ma che essa sciogle nel insieme delle manifestazioni umane.

$\mathrm{Ci}$ vuole precisare queste nozioni che stabiliscono le strutture basiche di una teoria antropossemiotica, apparti dei fenomeni zoosemiotici determinabili nei termini degli indici o dei segni naturali, ${ }^{3}$ anzittuto nel senso che l'intenzione è presente per la caratterizazione della fenomenica segnica come centro dell'attività di produzione dei segni. Non necessariamente intenzione di comunicare, però intenzione convenzionale di esprimersi. Quest'idea di convenzionalità è ammessa come diferenza centripeta perché sia possibile stabilire le diferenze tra le relazioni segnanali animali e quelle umane; l'uomo può esprimersi attraverso codici coligati ai

1. Nel contesto di questo saggio la parola segno verrà utilizata nel senso che gli atribuisce Peirce: "Un segno, orepresentamen, è qualcosa che, su certo aspetto ocerto modo, rappresenta qualcosa a qualcuno

2. Cfr. Umberto Eco, Struttura assente, 1989, p. 15.

3. La terminologia viene dell'utilizazione fatta da Eco nell'opera Il segno. 
fenomeni sociali che soffrono gli influssi dei valori venuti dalla società, e in questo senso, questi influssi sono le cause di trasformazione e perfezionamento del sistema di segni umani. Il dominio umano di un codigo, permette anche la costruzione artificiale di nuovi e sempre diversi significanti senza nessuna relazione intrinseca con la naturalità o con la empiricitità della realità oggettiva; delle catene significative si stabiliscono a partire delle manipulazioni umane del codigo primario di relazione soggetto cognoscente-realtà oggettiva. In questo senso, l'uomo è produtore di cultura e di modificazione dei fenomeni naturali, tra i quali si incontrano i fenomi signici.

Il fenomeno estetico preso dal punto di vista della teoria dei segni -- la chiamata semiotica ${ }^{4}$ dai teorici che studiano i segni e le loro relazione interne ed esterne -- e della prospettiva della culturalità della produzione segnica, intanto si possa parlare veramente di segni (pressuposizione di intenzionalità) e non propriamente di indici (l'intenzionalità è assente), permette l'oposizione di due materie distinte teoricamente e che hanno un certo contenuto di relatività $\mathrm{e}$ reciprocità cientifica: la estetica filosofica e la semiologia estetica. Così, per aprofondire queste nozione di rami teorici diversi si può introdurre la problematica attraverso le parole di Umberto Eco: "[Se] la estetica è la filosofia che specifica la propria attenzione sui problemi dell'arte e del bello, il campo degli studi estetici supera quello degli interessi semiologici, e una estetica semiologica è solo una delle possibilità dell'estetica; ma è certo che ne costituisci oggi una delle più feconde; mentre la considerazione semiologica può fornire lumi anche a chi affronti i problemi dell'arte da altri punti di vista filosofici (ontologia dell'arte, teoria delle forme, definizione del processo creativo, rapporti tra arte e natura, tra formatività naturale, tra arte e società, eccetera)" (1989: 408).

Partendosi delle indagini primigene verso l'indagine circa le questione di definizione del segno, quello che orienta la ricerca nel senso dellidentificazione dei suoi tipi, senza escludere di quest'analise la possibilità di tipificazione categoriche circa la natura dei segni ed i topici centrali i quali si riferiscono alla struttura dei fenomeni segnaletici. Così, dal principio, si può costruire il discorso sui segni, nel senso in cui si ha precisato la questione, stabilindo le prime nozione introdotte da Peirce come punto di partita di tutti i tipi di ragionamenti circa la

4. Il termine semiotica appartiene propriamente a una vertente caratteristica che viene da Locke, e ripresa da Peirce, e ha l'amplitudine della ricerca totale circa i fenomeni segnici. 
problematica segnico-culturale. Queste posture peirceane non lasciano di rappresentare una presa di posizione tendente a escludere la limitazione di un discorso che stabilisca la limitazione dell'analise dei diversi tipi di segni a quell'analise del segno esclusivamente linguistico. ${ }^{5}$ L'analise esclusivamente di carattere linguistico dimostra la capacità di vedere soltanto uno degli spazi di produzione segniche dentro l'universo di implicazioni dei fenomeni segnici; quest'ultima teoria dei segni linguistici è la scienza a cui si riferisce Saussure. ${ }^{6} \mathrm{Ma}$ la fenomenica non si incontra ristretta ai punti linguistici di riferenza, perché trascende queste possibilità restrite, ed anche restritive di stabilire significati e di trasmettere messaggi comunicativi, quantunque i segni linguistici siano veramente i più importanti dei veicoli del pensiero umano, attraverso i quali si possano interagire tutti gli uomini nell'espressività dei suoi pensieri.

La galassia della produzione segnica, non potendo essere ridotta alle considerazioni linguistiche, introduce la necessità di verifica delle prospettive in cui si può identificare la manifestazione culturale che interessi alla semiologia, o più propriamente alla semiotica. ${ }^{7}$ In fatto, i soggetti umani sono capaci di produrre dei segni, nel senso in cui stabiliscono codici di riferenza tra se stessi con la possibilità di trasmettere messaggi comunicativi veramente intenzionati, messaggi essi che sono presi dal ricettore che procede alla decodifica di tutta la struttura segnica comune ai parlanti -- ai praticanti del sistema di segni. Ma, non è soltanto l'intenzionalità che muove i processi comunicativi -- e qui si possono costruire considerazioni diverse circa i processi cognitivi e la relazione dell'uomo con la natura --, perché si possono risaltare relazioni di senso, di contenuto semantico, anche senza avere intenzionalità negli atti praticati dagli uomini. Questi due universi distinti corrispondono

5. 11 concetto peirceano di segno è più ampio di tutti gli altri concetti nel senso in cui può componersi di tutte le manifestazione che abbiano la caratteristica della sustittutività. Tutte queste considerazione si distacano della sua definizione, qualle sia: "Un segno, o representamen, é qualcosa che, su un certo aspetto o un certo modo, rappresenta qualcosa per qualcuno"

6. Per vedersi le diferenze tra un concetto di semiotica e di semiologia, come rami che discendono di teorie segnaletiche diverse che oponiscono Locke e Peirce a Saussure, consultarsi le note circa del problema in Eco, 1989: 383.

7. Qui facciamo le differenze tra semiologia e semiotica, nel senso in cui la prima sarebbe la scienza che studierebbe i segni linguistici, teoria saussuriana, intanto la seconda sarebbe la scienza che studierebbe la pluralità di tutti i segni, e non soltanto quelli linguistici, essendo questa la vertente peirceana di interpretazione dei fenomeni segnici. 
propriamente a quelli universi peirceani circoscriti in esfere diverse di attuazione, l'universo dei segni e l'universo degli indici. ${ }^{8}$

Circa la definizione degli indici, la natura, nella sua oggettività, al di là dell'universo dell'artificio umano, è anche un centro di produzione di messaggi indiziali, nel senso in cui la decodifica dei fenomeni, oggetti, processi ed anche degli altri riflessi dell'atuazione delle leggi naturali, porta l'osservatore a ritenere elementi per la costituzione di messaggi significanti, senza potersi parlare di intenzionalità del messagio. I codici naturali, o più propriamente la visione umana della fenomenica naturale, è importante in questo punto nel momento in cui si può distinguire la realtà intanto oggettività e la realtà intanto conosciuta dall' uomo. Gli indici sono stratti dal suo contesto naturale per comporre tutto un insieme di decodifiche proprie alle quale l'uomo atribuisci significati propri. In questo senso, il processo di conoscimento si inizia con la percezione dei fenomeni naturali, intanto tali, con tutta la visualità che gli è imanente, partendo, dopo, per lo stabilimento di relazioni che permettono i tipi di ragionamenti cientifici diversi, tra i quali, la deduzione, l'induzione, l'adbuzione e l'analogia. ${ }^{9}$ Con questi ragionamenti, e partendosi dalla riferenza necessaria della natura, sopra gli indici, si costruisce una esfera propria della cultura umana che si riferisce all'espressione dei sentimenti e alla creazione dello spirito umano che non può essere dissociata di tutti i suoi momenti di origine e di tutto l'insieme di considerazioni circa la manifestazione naturale indiziale. $\mathrm{L}^{\prime}$ esteticità culturale umana, dunque, partendo degli indici, costruisce tutta la struttura dei segni estetici, nel senso in cui, non soltanto come mímesis, ma come risultato di un processo di conoscimento umano, l'uomo è capace di costruire gli universi della sua saggezza sopra la dialettica esistente tra la decodificazione degli indici e la codificazione dei segni. ${ }^{10}$ Tra questi segni codificati, emergono principalmente quelli chiamati visivi.

8. Per la concettuazione di segni ed indici nella teoria peirceana, si può ritenere la relazione esistente tra gli indici e gli altri due elementi della tripartizione dei segni in rapporto all'oggetto, il simbolo e l'icone.

9. Questo appartiene propriamente a una teoria del conoscimento che fa la ricerca dei processi di apreensione e di lavoro mentale sui datti della realtà.

10. Questa dialettica appartiene alla struttuta umana di formazione della cultura; questo processo di ricostruzione razionale della natura e il passaggio delle strutture degli indici ai segni è propriamente quello che si può risaltare come importante per la ricerca della genetica dei segni. 


\section{La formazione dell'opera estetica}

Dal mondo della naturalità al mondo dall'artificialità della cultura umana c'è proprio questa fase di trasporto degli elementi della natura per quelli altri del pensiero. La noeticità della conoscenza ed i suoi risultati trasformativi nel operare l'oggettività costituisce propriamente lo spazio del costruire umano, della produzione costrutivo-trasformazionale della ragione umana. La pittura, per esempio, intanto insieme di relazioni di colori, sistema di riferimento che ha un codigo proprio, un canale proprio, un messaggio pittorico, è la tipica manifestazione del processo di separazione, individuzione e raccolta di fenomeni naturali visivi, per la composizione di un testo semiotico. ${ }^{11}$ Tutte queste riferenze non possono, dunque, essere dimenticate quando si procede allidentificazione della comunicazione tra le diverse forme di manifestazione artistiche ed estetiche umane.

$\mathrm{Ma}$, queste considerazione non sono state fatte senza un motivo centrale, motivo questo che consente osservare la problematica estetica con l'aiuto di premesse varie, tra le quale, quelle di carattere semiotico. In questo senso la domanda a essere fatta è la seguente: quando si può considerare un messagio come una costruzione estetica? ${ }^{12}$ Per rispondere al questionamento furono presentati tantissime risposte, ed anche tantissime scelte ideologiche e filosofiche furono utilisate nel senso di presentarsi una conclusione sulla tematica che è sempre motivo di dibati e di contende tra gli intelletualli e i teorici dell'arte e della filosofia estetica. In questo punto bisogna rimmettere la questione in termini di analise comunicazionale, e, in questo senso, si può costruire un modelo che si basa sul piano delle funzione dei messagi nel insieme delle considerazioni sull'universo concettuale della comunicazione umana. Normalmente, come tipi di messagi si possono sottolignare i messaggi referenziali, poetiche o apelative, fatiche, metalinguistiche, imperative ed estetiche, e attraverso questo modelo di partizione di definizione didascalica iniziare un'altro indagine di carattere più specifico sulla manifestazione

11. Per una nozione sul concetto di testo semiotico si deve ritenere che tutti i composti di segni che hanno la caratteristica di essere ambigui e semioticamente complessi possono essere chiamati di testi semiotici.

12. "Nell'ambito della terminologia filosofica italiana, quando si parla di estetica, si intende una indagine speculativa sul fenomeno arte in generale, sull'atto umano che lo produce e sulle caratteristiche generalizzabili dell'oggetto prodotto" (Eco, 1993: 185). 
estetica. ${ }^{13}$ Nell'ambito di questo saggio, il riferimento principale si farà intorno a quest'ultimo tipo di funzione del messagio, l'estetico, nonostante sia importante dire che non è possibile vedere questo come un quadro dogmatico, anzi flessibile, davanti alla complessità delle emizioni comunicazionali del quotidiano, che non si utilizano di un o di altro tipo di funzione, ma si costruiscono dalla coligazione di molti di questi.

La scelta per l'usuario delle struture segniche di comunicazione per trasmettere i suoi desideri, i suoi costrutti teorici, i suoi valori ed altri contenuti intenzionali che rimangono nell'ambito del sottocodice, non è qualcosa di arbitraria; obedisce a tutti i rapporti logici esistenti nella struttura di questo sistema non organizatto, ma organizabile di un repertorio di segni che significano nelle relazioni sociali. A priori, l'entropia ${ }^{14}$ dei segni del sistema non permette la distribuzione perfetta del repertorio di elementi, siano linguistici, siano plastici, siano musicali, siano pictorici, lavoro che rimane a cura del impegno razionale dell'uomo nella modificazione della attività di plasmare la sua partecipazione nell'insieme di elementi naturali. Il messagio estetico, non è, in questo senso, una qualsiasi scelta di segni di un sistema di segni, ma una scelta propria per la costituzione di un sistema individuale di significazione, a partire del impiego dei segni forniti dalla struttura codificata. Adesso si permette introdurre la nozione che c'è il suo cabimento perfetto in questa parte della discussione: la nozione di idioletto, nel senso in cui la parola è ammessa come significante per Umberto Eco. Il riferimento all'idea di idioletto come "(...) il codice privato e individuale di un solo parlante (...)" (Struttura Assente, 1989: 68), non introduce niente di diverso ni di quello che si viene dicendo nelle linee di sopra, neanche di quello che il senso comune sa sulla tematica, o sia, che l'usuario del sistema di segni, nel momento di costruire un insieme di messagi significanti non lascia di utilisare dei suoi atributti di arbitrarietà e di creatività, di forma a creare la sua stessa forma di utilisare, diversamente di tutti gli altri, le

13. Queste considerazioni concettuali si riferiscono alla classificazione didascalica della teoria di Jackbson, quello che diventò già una tradizione acettata da tutti come modelo teorico sui problemi del messagio.

14. Per il concetto di entropia: "L'informazione misura dunque di eqüiprobabilità, di distribuzione statística uniforme che esiste alla fonte; e questo valore statistico è quello che $\mathrm{i}$ teorici dell'informazione, desumendolo dalla termodinamica, chiamano entropia.". E Anche: "L'entropia è altrimenti identificata con uno stato di disordine, nel senso in cui l'ordine è un sistema di probabilità che si introduce nel sistema per potere prevedere l'andamento" (Eco, La struttura assente, 1989: 25/ 26). 
premesse del sistema strutturato di segni. Il risultato è chiaro agli occhi di tutti: il subsistema significante dell'autore è un sistema capace di costruire istrumenti particolari di manipulazione della struttura segnica, senza romperla, ma, neanche, senza obedirla; l'asttrazione è fatta delle norme del sistema, perché delle nuove sono formate, e esse sono regole individuali, che hanno un alto senzo di ambiguità e di autoriferimento. Queste due caratteristiche sono sostanzialmente l'essenza di tutto quello che può dire sul messagio estetico. La esteticità di un messagio è tanto più acentuata quanto più forte siano i riflessi dell'ammissione di queste due caratteristiche per la formazione del idioletto.

L'idioletto traduce l'idea centrale del processo di lavoro culturale ed estetico, o sia, la creazione, e, in questo senso, l'attività creativa non può venire considerata semplicemente come quell'attività di riunione di un repertorio di oggetti caoticamente racogliti dal mondo reale; la creazione non è soltanto quel insieme organizzato di materie prese dalla realtà oggettiva. L'atto di creazione ̀̀ propriamente la costruzione di una complessità significativa ed il significare importa per forza in un esercizio di selezione e ordinazione di segni -- e quest'ordinazione chi l'ha fatta è stato il produttore dell'opera estetica, con certi gradi di ambiguetà e di autoriferimento di modo a costituirsi lidea di completude del messagio estetico --, siano questi segni simbolici, siano iconici, siano indicativi, secondo la tripartizione peirceana. L'ordine di un testo è l'ordine della volontà di espressione estetica del creatore; non è nient'altro che l'ordine desiderata dall'autore. L'insieme di segni è soltanto l'istrumentazione di quella volontà di espressione intuitiva, sentimentale, di gusto e di piacere, colligati alla capacità umana di manipulazione del significante segnico.

Di solito, le prime diferenze sono già lineate come parametri di orientazione per la discussione del problema centrale e primario attorno alla concettuazione di opera estetica, tanto come attorno alla depurazione della nozione di esteticità dell'insieme di considerazioni culturali che si possono prendere dell'universo dell'artificio umano. Però si trova un'altro punto da chiarire, essendo questo punto qualcosa di molto importante nel contesto di questa discussione: il problema delle relazione tra teoria e pratica dell' estetica. Si di una parte ci sono elementi teorici per la composizione di idee sull'arte, e non propriamente di arte in se stessa, dell'altra parte c'è propriamente sua pratica, intanto attività tecnica di costruzione e elaborazione di fenomeni estetici palpabili e sfruttabili. 


\section{La estetica ed i segni estetici}

Ma quello che bisogna essere chiarito in questo punto è che la nozione di estetica, come problema filosofico, e come teoria circa i fenomeni artistici, non c'è niente, o c'è poco, da vedere col proprio sviluppo della nozione di arte. Infatti, due universi di valori distinti, che si fondono in radici diverse, non possono essere confosi. La teoria estetica e le considerazioni dello spirito circa i problemi estetici non hanno le stesse motivazioni delle pratiche artistiche in generale. Nelle parole di Croce non si può parlare veramente in una teoria estetica di carattere filosofico prima del Seicento, perché c'è una "(...) simultanea nascita della filosofia moderna e dell'Estetica. L'origine di questa si colloca infatti, come si è acenatto, tra il Sei e il Settecento, ossia coincide con la nascita del <<soggettivismo>> moderno, con la filosofia come scienza dello spirito (...)" (Croce, 1992: 130). Intanto, l'istoria dell'arte radica i primi pensieri sull'arte nello stesso momento in cui l'uomo a cominciato a producerla; questa come risultato non può divergere del suo intendimento sui fenomeni reali. Quello che veramente è accaduto è che la ragione umana si è sviluppata intensamente introducendo una necessità di depurazione teorica più perfezionata per capire ed interpretare i fenomeni estetici. ${ }^{15}$

La propria nozione di estetica come teoria che propone l'esame di tutte le forme di manifestazione segniche che possano trasporre i limiti della coscienza dell'autore e formare un insieme culturale capace di trasmettere dei messaggi intenzionati fornisce degli elementi per la presentazione di questo problema. La formazione dell'opera è un processo più complesso di quanto si possa imaginare quello che la fruisce, perché tutta l'attività estetica si distribuisce del primo momento di conoscenza della realtà fino alla depurazione delle sue imagine, e forme, e alle attività di materializazione delle forme.

Le forme in cui si manifestano i fenomeni estetici dipenderà di tutto un insieme di considerazione che vanno dalla volontà del artista alla proprietà delle materie ad essere lavorate (materie plastiche, sintetiche, colori, suoni, luci...). Ma, la

15. In altre prospettive, per capire le diferenze essenziale in questo punto si possono prendere in considerazione altri elementi di riferenza, qualli siano le relazioni tra religiosità, mitologia, filosofia e esteticità culturale. Secondo Croce: "Ma la distinzione dell'arte della filosofia (intesa questa nella sua ampiezza, che comprende ogni pensamento del reale) trae seco altre distinzioni, delle quali, in primo luogo, quella dell'arte del mito." (...) "All'arte, per essere mito e religione, manca appunto il pensiero, e la fede che da esso si genera; l'artista non crede e non discrede la sua immagine: la produce." (Croce, Breviario di estetica, 1992: 32). 
formazione del segno sarà sempre un'attività di produzione e di distribuzione di queste materie secondo forme primigine del pensiero segnaletico del creatore. Così si può vedere l'amplitudine in cui si manifesta lo spazio culturale della produzione di segni estetici, perché questi non sono soltanto i segni risttreti a uno dei sensi umani, ma a tutti quelli che componiscono l'uomo nella sua totalità di percezione. Queste conclusione si distacano del proprio concetto di estetica, che, etimologicamente, nella sua radice greca (aísthetos), si rapporta a tutti i fenomini sensibili, in oposizione a quelli noetici, o prodotti nel chiuso universo delle manifestazione intelletive. La sensibilità umana non è soltanto visiva, ma anche auditiva, tattuale, gustativa, olfativa, e è in questo senso più generale che si può fermare le basi della manifestazione estetica sui diversi piani della sensorialità umana, con segni propri per la stimulazione di ogni livello della sensibilità.

La formazione dell'opera ad essere fruita deve ocorrere come fenomeno fattico di reificazione o di formazione materialistica della creazione noetica dell'artista. Questo è necessariamente così perché "[Non] c'è arte (...) se non interviene un oggetto fisico, un'opera elaborata con criteri tecnici, più o meno specializzati e faceti capo a una professionalità riconosciuta" (Barilli, 1989: 85). Dunque, non soltanto intenzionalità e ni soltanto professionalità ripetitiva, ma la congiunzione di questi passi è necessaria per l'avvento dell'opera estetica come tale. In un primo momento c'è luogo il sentimento, l'intuizione, l'idea ${ }^{16}$ quel colpo mentale che darà origine all'espresione fisica della esteticità. ${ }^{17}$ L'arte senza sentimento non è arte, è soltanto la formalizazione di una sttrutura segnica già ammessa come significante tra i partecipi di una comunità culturale.

"Il problema non è di evitare comunque le emozioni, ma di farle nascere appunto, in uno spazzio controllato (...)" (Barilli, 1989: 164), quello che vuol dire che la manifestazione richiede delle forme esteriore per poter essere manifestata e intesa da tutti gli altri uomini che la possano fruire. Le forme richiedono, anche, la partecipazione dei segni nella loro composizione, segni questi

16. Il termine idea l'usiamo qui come nel suo uso originale della parola greca idea. Non si tratta semplicemente di un ritorno alle nozione primigine della parola, ma anche di un'aprensione più concreta del senso del termine; idea significa proprio quel primo momento di luce che aparisce nel pensiero del creatore come punto di partita per i gradativi processi di formalizazione e materializazione della cosa creata.

17. "L'artista, dunque, quasi per definizione deve emozionarsi, essere sensibile ad aflussi energetici di ordine affetivo-passionale, più di quanto non capiti al comune mortale" (Barilli, 1989: $150 / 151)$. 
propri a ogni tipo di riferimento che l'artista voglia costruire. Quest'analise dei fenomeni segnaletici, ed anche della genetica dei fenomeni mimetici dell'uomo sulla natura, non costituisce nient'altro che un punto di partita per l'introduzione delle questione più precise circa la problematica di definizione dei segni estetici.

L'enunciazione del momento estetico come un momento culturale chiede la definizione di quale siano questi prodotti culturali che non soltanto partecipano della vita sociale, ma che anche hanno i traci diversi della culturalità espressiva di sentimenti estetici, traci questi capaci di comporre l'organicità e l'unicità tra tutte le manifestazione dite estetiche. Così, con Barilli (1989: 58/75) si possono sottolineare i simboli estetici come: linguistici, quelli che corrispondono ai diversi segni utilisati dal linguaggio, siano orali, siano scritti (gli alfabeti, le scritture); musicali, come essendo tutti i segni che si utilisano per l'affetazione del senso auditivo umano tra i diversi codici musicali (le note nelle sue espressione visive o auditive, sistema sonoro e notativo); grafici, come quelli che si risalgono per la sua visualità, o sia, per la sua iconicità ${ }^{18}$ (pittura, scultura e archittetura). ${ }^{19}$

Un segno estetico, in questo senso, sarà propriamente quello che possa rappresentare una forma segnica capace di esprimere la volontà del creatore. Ormai sono dentro questi tipi di manifestazione culturale quelle scientifiche, quelle litterarie, quelle artistiche, in un senso generale. Non si può chiudere l'universo estetico tra le considerazione uniche della univocità espressiva, anzi il complesso ruolo che i segni svillupano nella formazione del proprio pensiero umano richiede l'attenzione del teorico della estetica nel senso di determinare quall'è, o qualle possa essere l'amplitudine totale del galassia segnica a essera scoperta o assinalata perché si possa parlare di una teoria totalizante dell'insieme estetico. Tutti i segni segnalati hanno come proposta di intendimento estetico le caratteristtiche della plurivocità, fruibilità, segnicità.

18. Tra i diversi segni naturali visivi, così chiamati da Eco, si possono rilevare i segni iconici.

19. Anche ci sarebbero altre forme di vedere i fenomeni estetici attrarverso diverse altre partizioni didascaliche, come tra i segni dello spazio (pittura, architettura) e dello tempo (musiche, canzione, opere litterarie). Un'altra proposta sarebbe questa: "Comunque, appare qui una biforcazione naturale tra opere d'arte bidimensionali (pittura, disegno) e invece opere tridimensionali (scultura), con la possibilità di numerosi generi intermedi e misti (bassorelievi, oggi anche collages, assemblages, <<ambienti>>) "(Barilli, 1989: 108). 


\section{Aspetti interpretativi dell'opera estetica}

La partecipazione del fruitore nella composizione dell'opera stetica permette proprio la formazione di un concetto aperto del atto di creazione artistica. ${ }^{20}$ Tutti i costruti culturali umani si referiscono alle diverse possibilità di interpretazione del significato della estetica nel opera contenuta, in modo che, qualsiasi che sia l'interprete dell'opera artistica, nuovi prospettive di significato saranno aperte. Quindi, la dificoltà di determinazione degli effeti propabili dell'opera nel contesto della culturalità umana ci riporta all'idea di una catena di riflessi non misurabili tra le diverse generazioni; l'opera estetica è propriamente un oggetto culturale, e come un oggetto culturale, si fa presente tra gli uomini ad infinitum.

Il mondo ermetico e psicologico del creatore definisce il senso intenzionale iniziale dell'opera, quello che non impedisce che produsca sul piano dell'alterità dei diversi tipi di risultati interpretativi. ${ }^{21}$ Quando l'opera non è più chiusa nell'idealità -- universo delle idee creative del autore del messagio estetico che componisce il piano noetico umano --, e aviene alla realtà del mondo sensibile come oggetto materiale -- lasciando di essere pensiero e tornandosi veramente un'opera --, perde sua caratteristica di ingegno particolare e diventa elemento culturale apropriabile per qualsiasi persona che partecipe dell'opera o della fantasia artistica di creazione e interpretazione. È veramente in questo senso che si può parlare di un opera in movimento. ${ }^{22}$ C'è qui pertinenza l'introduzione della riferenza alla nozione di opera estetica come quella che è il risultato della attività di creazione, manipulazione segnica, elaborazione e perfezionamento della materia nella cui si espressa, in modo a costituire uno spazio culturale che ha un'ordine interno proprio -- che corrisponde all'idea di idioletto dell'autore --, appropriabile e

20. Si può prendere la nozione di testo, data da Eco, per vedere la partecipazione dell'interprete nella formazione dei sensi di un'opera qualsiasi: "Un testo vuole che qualcuno lo aiuti a funzionare" (Eco, Lector in fabula, 1989: 52). E anche: "(...) un testo è un prodotto la cui sorte interpretativa deve far parte del proprio meccanismo generativo (...)" (54).

21. "(...) l'autore ha certo il diritto di pronunciarsi sulla sua opera, di dotarlo di un congruo pacchetto di istruzioni per l'uso, che ne sono parte costitutiva, e che pongono anche al lettore l'obbligo di prenderne conoscenza; ma un tale diritto a sua volta non vincola il diritto del fruitore di esercitare le prerogative sancite proprio delle teoria estetica, per cui l'opera è un contesto organico, polisemico, <ambiguo>, dove quindi è possibile scoprire associazioni, rimandi, collegamenti, di cui l'autore non era consapevole" (Barilli, 1989: 169).

22. Cfr. Umberto Eco, Opera aperta, 1993. 
manipulabile per qualsiasi persona che venga a fruire come spetattore attivo dell'opera prodotta.

L'opera che si fa in movimento $^{23}$ è qualsiasi creazione spirituale umana che possa essere definita in acordo con le nozione di esteticità e di costruzione artistica. L'autenticità del codice individuale stabilito dall'autore del insieme di relazioni di segni, di significati e di di riferenze alla realtà o ai diversi livelli di significazioni $^{24}$ delle funzioni dei messagi della comunicazione (referenziale, se l'autore si riferisce alla realtà attraverso un giudizio di affermazione o negazione; apelativa, se l'autore vuole ridurre la distanza tra se stesso e il lettore o fruitore, lo facendo vivere diretamente le suttilileze del messagio estetico, etc.).

Così, chissà, attraverso questi apuntamenti semiotici, culturali, ed estetici si possano distrurre le vechie nozione dicotomiche che ancora sopravivano nel concettuare i diversi tipi di arte e nel formulare giuduzi estetici sulle diverse forme di esporre la sentimentalità creativa dell'autore. Una di queste dicotomie è propriamente quella, ben notta da tutti, che stabilisce una diferenza tra opere classiche e opere moderne; le opere classiche e le moderne, posmoderne o contemporanee, sono tutte sottomesse alle regole della diacronicità, della dinamica di vita dell'opera artistica e estetica, che si trasforma a ogni momento fruizionale diverso, tanto a causa del cambiamento di fruitore, come a causa della distanza temporale tra le fruizioni di uno stesso osservatore dell'arte. L'unificazione di tutti i tipi di fenomeni estetici sotto le considerazione dell'interpretatività del costruto semiotico e della libertà del fruitore dell'opera può formare le nuove ligne della teoria estetica fino ad oggi considerata. In questi termini, si può seguire le considerazione di Eco sulla tematica, che dice che "(...) qualsiasi forma d'arte, anche se adotta le convenzioni di un discorso comune o simboli figurativi accettati dalla tradizione, fonda il proprio valore su una novità di organizzazione del materiale dato che costituisce in ogni caso un aumento di informazione per il fruitore. Ma attraverso a impennate originali e roture provvisorie dell'ordine delle previsioni, un'arte classica mira in fondo a riconfermare le strutture accettate dalla sensibilità comune a cui si

23. "(..) anche nell'affermarsi di un'arte della vitalità, dell'azione, del gesto, della materia trionfante, della piena causalità, si stabilisce una dialettica ineliminabile tra opera e apertura delle sue letture. Un'opera è aperta sinché rimane opera, oltre questo limite si ha l'apertura come rumore." (Eco, Opera aperta, 1993, p. 177).

24. "Il simbolo artistico ha proprio il compito di selezionare un ampio spettro di significati" (Barilli, 1989: 67). 
rivolge, opponendosi a determinate leggi di ridondanza solo per riconfermarle di nuovo, sia pure in modo originale. Invece l'arte contemporanea pare perseguire come valore primario una intenzionale rottura delle leggi di probabilità che reggono il discorso comune mettendone in crisi i pressuposti nel momento stesso in cui se ne avvale per deformarlo" (Eco, 1993: 168-169).

São Paulo, dezembro de 1995.

\section{BIBLIOGRAFIA}

CROCE, Benedetto. Breviario di estetica. 2a edizione. Milano, 1992.

$\mathrm{ECO}$, Umberto. Opera aperta: forma $e$ indeterminazione nelle poetiche contemporanee. Milano : Bompiani, 1993.

ECO, Umberto. La struttura assente. Milano : Bompiani, 1989.

ECO, Umberto. Lector in fabula. Milano : Bompiani, 1989.

BARILLI, Renato. Corso di estetica. Bologna : Società Editrice Il Molino, 1989. 